\title{
Monkey virus may be cleared of cancer link
}

Helen Pearson, New York

Laboratory contamination could have lent unwarranted support to the contentious idea that a monkey virus causes certain types of cancer, according to a study published last week.

The study tackled a long-standing disagreement in cancer biology about whether simian virus 40 (SV40), which contaminated stocks of polio vaccine in the 1950s and 1960s, could have infected vaccinated patients and triggered a range of chest, bone, brain and blood cancers. In support of this idea, some laboratories have identified fragments of the virus in tumour tissue samples - but others have struggled to repeat their results. The new study, led by pathologist Marc Ladanyi of the Memorial SloanKettering Cancer Center, New York (F. López-Ríos, P. B. Illei, V. Rusch and M. Ladanyi Lancet 364, 1157-1166; 2004), suggests a reason for this discrepancy.

"It's a very major finding and may resolve the controversy," says microbiologist Keerti Shah of the Johns Hopkins Bloomberg School of Public Health in Baltimore, Maryland, who has argued against a link between the virus and cancer. The study suggests that other researchers investigating the question may have inadvertently contaminated their

motion of galaxies, an effect normally attributed to the presence of dark matter, but physicists have pointed out that it could also account for the Pioneer anomaly.

Anderson now wants to help resolve the uncertainty by reanalysing data from the first decade of the Pioneer missions. Slava Turyshev, a colleague of Anderson's at JPL, estimates that it will cost about US\$250,000 to fund the analysis, and a grant application will be submitted to NASA later this year.

Turyshev and colleagues also have a grander plan: a dedicated spacecraft that would follow a similar trajectory to the Pioneer missions in a bid to recreate the anomaly. Their proposal, outlined in Paris on 16 September to an advisory panel of the European Space Agency (ESA), involves launching a spacecraft that would be followed a few kilometres behind by a reflective ball. Lasers on the craft would monitor the distance between the ball and craft, allowing researchers to detect and compensate for any acceleration caused by events on the craft, such as heat leaks.

But the craft would cost at least US\$500 million, and sources close to the panel suggest that it will not make the project one of the two priorities that it must convey to ESA next month.

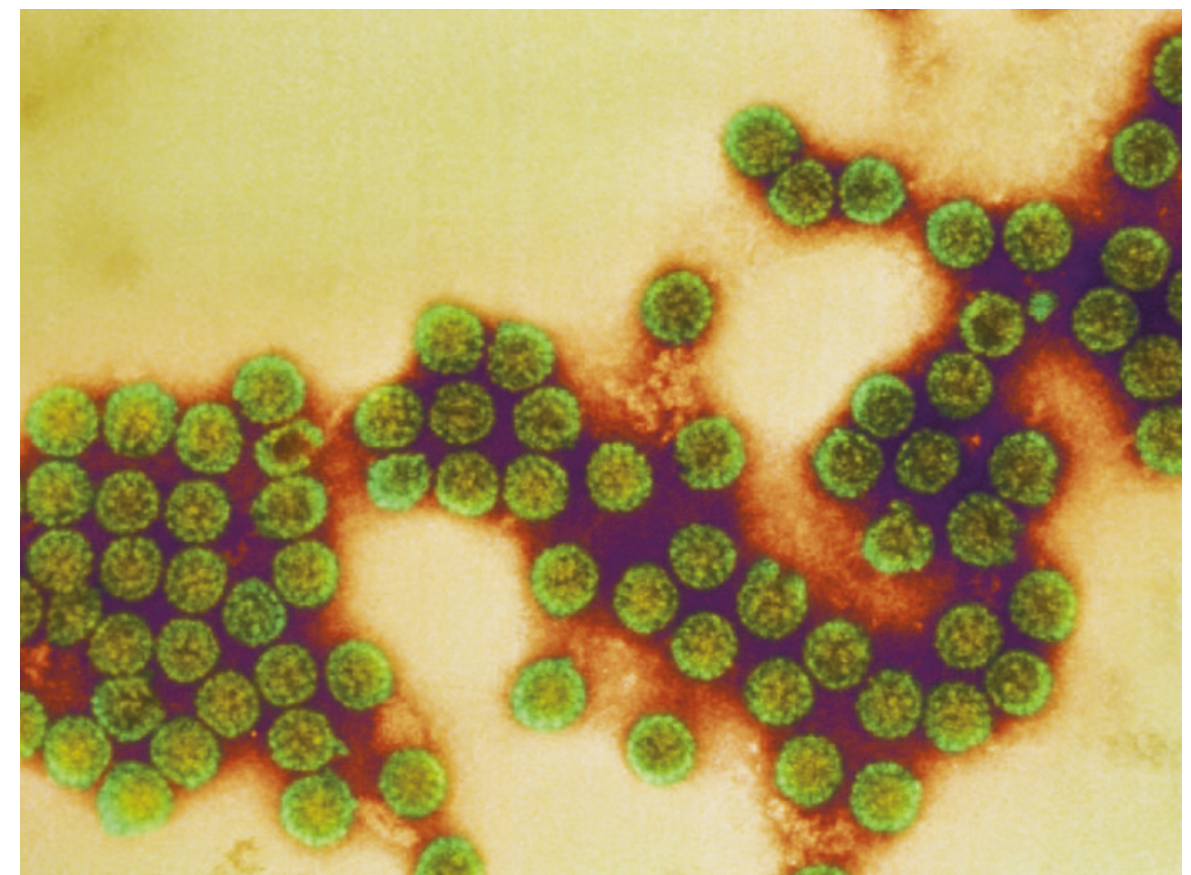

Simian scapegoat: some still argue that this monkey virus caused cancer through a contaminated vaccine.

tumour samples with plasmids - pieces of DNA that are widely used in molecular genetics laboratories and that frequently contain hidden fragments of SV 40.

Ladanyi and his team searched for the virus in tissue samples of mesothelioma, a type of chest cancer. They used the polymerase chain reaction (PCR) to amplify a small stretch of DNA from the virus, and initially found that it was present in around $60 \%$ of their samples. But when they compared the genetic sequence of this region with others in gene databases, they realized that the section was also found in numerous laboratory plasmids.

Ladanyi and his team then carried out a series of detailed experiments showing that the virus DNA they had detected had come from contamination with plasmids, rather than the intact virus. Using genetic sequencing, for example, they showed that the virus fragment present in their samples contained a genetically engineered gap that is only found in plasmids.

Ladanyi believes other laboratories may have overlooked similar contamination in their PCR experiments, because they were unaware that so many plasmids contain sequences from SV40. To back up this argument, the team reanalysed the results of a 2002 study that identified the virus in brain and bone tumours (F. Martini et al. Cancer 94, 1037-1048; 2002). The viral sequences this group had published showed that it too had detected plasmid sequences rather than SV40 itself.

The onus now falls on other laboratories to re-examine their results and rule out contamination, says cancer epidemiologist Eric Engels, who studied the issue at the National Cancer Institute in Bethesda, Maryland. "Laboratories need to take this result seriously," he says.

But others in the field who have detected the virus in their cancer samples stand by their original results. Virologist Janet Butel at the Baylor College of Medicine in Houston, Texas, says that her group has carefully checked its samples for plasmid contamination and can confidently rule it out. "This experiment does not mean all other laboratories are similarly contaminating their samples," she says.

Those on both sides of the argument have other evidence to support their case. If the vaccine caused cancer, there should have been a rise in mesotheliomas or other cancers among those vaccinated decades ago with the virus-contaminated jab. But epidemiological studies have not detected one. On the other hand, hamsters and rats injected with SV40 go on to develop tumours.

Because the two sides are so entrenched, this single study is unlikely to resolve the debate, predicts Chris Wilson, an immunologist at the University of Washington in Seattle. Wilson served on a 2002 National Academies panel that assessed the evidence linking the virus and cancer. Either way, the study highlights the perils of PCR, which is notorious among researchers for producing false results in a variety of situations, even with the most experienced groups. "We know there are pitfalls," says Wilson. "and one has to be extremely diligent to avoid them." 